\title{
Partial deletion of the Saccharomyces cerevisiae GDH3 gene results in novel starvation phenotypes
}

\author{
Barrie M. Wilkinson, $\uparrow$ Carolyn M. James and Richard M. Walmsley
}

Author for correspondence: Richard M. Walmsley. Tel: +44161200 4174. Fax: +441612360409.

e-mail: walmsley@umist.ac.uk

Department of

Biochemistry and Applied

Molecular Biology,

University of Manchester

Institute of Science and

Technology, PO Box 88,

Manchester M60 1QD, UK

\begin{abstract}
A small-scale functional analysis screen has revealed several new phenotypes associated with a large deletion of GDH3, one of two Saccharomyces cerevisiae genes known to encode NADP-linked glutamate dehydrogenase. Diploids heterozygous for the deletion are able to sporulate in rich media, while haploid deletants produce dark, wrinkled colonies containing pseudohyphal cells. The haploid cells rapidly lose viability upon starvation.
\end{abstract}

Keywords: $G D H 3$, polyGT, foraging, nitrogen metabolism, starvation

\section{INTRODUCTION}

Since the completion of the genomic sequencing of Saccharomyces cerevisiae it has become evident that there is a surprising amount of genetic redundancy in this very small genome. However, it may be that much of this redundancy is more apparent than real (Oliver, 1996), and that very similar genes may have distinct physiological roles to play within the yeast cell. For instance, there are two genes encoding citrate synthase. However, CIT1 (on chromosome XIV) encodes a mitochondrial enzyme, whereas CIT2 (on chromosome III) encodes the cytoplasmic (probably peroxisomal) enzyme (Rosenkrantz et al., 1986, 1994). Such specialization of function can sometimes be recognized from an analysis of the sequence (in this example, by the recognition of a mitochondrial targeting sequence). However, in many instances, the distinctive roles of very similar proteins will only be revealed by careful experimental phenotypic analysis of strains carrying mutations in the different genes of an apparently redundant set.

In yeast, the interconversion of ammonia and glutamate forms the interface between the biosynthesis and degradation of nitrogenous compounds (Cooper, 1982). In anabolic processes, NADP-linked glutamate dehydrogenase (NADP-GDH) catalyses the reaction between ammonium ions and $\alpha$-ketoglutarate to form L-glutamate.

† Present address: Department of Cell and Molecular Biology, Stopford Building, University of Manchester, Manchester M60 1QD, UK.

Abbreviations: CHEF, contour-clamped homogeneous electric field; NADP-GDH, NADP-linked glutamate dehydrogenase; polyGT, polydGdT. polydCdA.
The principal phenotype of mutants deficient in this activity is a lowered growth rate when grown on ammonia, cytosine or urea and a failure to grow on allantoin (Grenson \& Hou, 1972; Cooper, 1982). No developmental abnormalities have been reported in these mutants. There are at least two yeast genes which encode NADP-GDH. The first, GDH1, was defined by isolating mutants which showed the aforementioned deficiencies in nitrogen assimilation. This gene has been cloned by Moye et al. (1985), using complementation of the corresponding lesion in Escherichia coli, but has never been genetically mapped. A second gene, GDH3, was discovered in the course of the systematic DNA sequencing of chromosome I (Bussey et al., 1995). The two genes show $86 \%$ nucleotide sequence identity in their coding regions, and a corresponding similarity at the level of the amino-acid sequence of their products. A third gene, GDH2, specifies the NAD-linked catabolic glutamate dehydrogenase, an enzyme which shares no significant amino-acid sequence homology with either Gdh1p or Gdh3p (BLASTP alignment; Altschul et al., 1990).

In the course of a programme of work designed to examine the phenotypic consequences of deleting internal polydGdT.polydCdA (polyGT) tracts from yeast chromosomes (Wilkinson, 1991), we fortuitously isolated a number of mutants carrying deletions at the $G D H 3$ locus (which lies close to a polyGT tract on chromosome I). An examination of the phenotype of these deletants led to the results presented here which shed new light on the relationship between nitrogen assimilation and the morphology and growth habit of S. cerevisiae (Gimeno et al., 1992). The study has also allowed us to assign $G D H 1$ to chromosome XIV. 


\section{METHODS}

Micro-organisms. Yeast strains used were $S$. cerevisiae MGG3, an isogenic diploid, $M A T \alpha / M A T$ a ura3-52 ura3-52 leu2-3,112 leu2-3,112, bis3D1 bis3D1 can1-100 can1-100 (Goebl \& Petes, 1986); strain MGG3-D34, a diploid derivative of MGG3 which has had a single allele of $G D H 3$ substantially deleted and replaced with the yeast $U R A 3$ gene (see Results); strain A364a, a haploid $M A T$ a ade 1 ade 2 ura 1 lys 2 tyr 1 bis 7 gal1; and strain SJR1Ya, a $M A T$ a haploid derivative of MGG3. Strain YP148 (MATa ura3-52 lys2 ade1 bis7 trp1; chromosomes VII and XV have each been made into two smaller chromosomes to facilitate their resolution in pulsed-field gels) was kindly provided by $\mathrm{P}$. Hieter of John Hopkins University, Baltimore, MD, USA.

E. coli strains used were JA221 ( $\operatorname{rec} A$ leuB6 $\operatorname{trpB6}$ bsdR $b s d M$ lac $Y$ ) and TG2 [rec $A$ thi supE bsdR $\Delta($ lac-pro $A B) \mathrm{F}^{\prime}$ (traD36 pro $A B^{+}$lac $I^{\mathrm{q}}$ lac $\left.\left.Z \Delta \mathrm{M} 15:: \operatorname{Tn} 20\right)\right]$.

Yeast growth and genetic analysis. Media, culture conditions and methods for mating and sporulation of $S$. cerevisiae were as described by Sherman et al. (1982). Yeast chromosomes were prepared by the method of Gardner et al. (1993) and separated by contour-clamped homogeneous electric field (CHEF) electrophoresis (Chu et al., 1989). Yeast Nitrogen Base (YNB, without amino acids and nitrogen; Difco) was used as the basal minimal medium in preparation of other supplemented media. Ammonium sulphate $(0.5 \%, \mathrm{w} / \mathrm{v})$ was added to $\mathrm{YNB}$ as a nitrogen source, except where noted differently in the text. Yeast Extract Peptone (YEP) medium was used with the following supplements : $2 \%(\mathrm{w} / \mathrm{v})$ glucose (YEPD), $2 \%(\mathrm{v} / \mathrm{v})$ glycerol (YEPG), $2 \%(\mathrm{w} / \mathrm{v})$ potassium acetate (YEPA).

Recombinant DNA techniques. Enzymes used in molecular cloning were used exactly as recommended by the suppliers. DNA sequence analysis was carried out using a Vistra DNA Labstation and an Applied Biosystems 373A automated sequencer. Other techniques were performed as described by Sambrook et al. (1989). A pUC18-derivative, lacking the single EcoRI site, was constructed by first cutting the plasmid with EcoRI, then filling the recessed ends using T4 DNA polymerase, before re-ligation.

In a previous study of yeast telomeres (Walmsley et al., 1984), a collection of polyGT-hybridizing clones was isolated from a YEP24 library of yeast genomic DNA fragments generated by partial digestion with Sau3A. The library contained single copy chromosomal polyGT sequences as well as clones of telomeric DNA because the telomeric polydG $\mathrm{di}_{1-3} \mathrm{dT} . \mathrm{dC}_{1-3} \mathrm{dA}$ repeat also hybridizes to polyGT (Walmsley et al., 1984). In this study we particularly wanted to study bona fide polyGT tracts, so telomere clones were identified and excluded from the collection. To achieve this the clones were prepared for colony hybridization and re-screened with two telomere probes prepared from the plasmid Yrp120 (Walmsley et al., 1984): a SalI-SphI fragment corresponding to the highly conserved $\mathrm{Y}^{\prime}$ repeat, and a HpaI-Sall fragment corresponding to the less highly conserved $\mathrm{X}$ repeat. The telomeric clones identified in this way were excluded from the study. The remaining polyGT-hybridizing clones were physically mapped using restriction endonucleases and, using this information, wholly internal polyGT-containing restriction fragments of the insert were removed and replaced with the selectable marker gene $U R A 3$ in vitro. Following this modification, the insert DNA was released with restriction enzymes and used to transform the isogenic diploid strain,
MGG3. A diploid strain was used in the transformation experiments because we expected to find some deletions that were recessively lethal. The transformants were sporulated and the resulting asci were dissected on rich medium (YEPD) prior to further analysis.

Microscopic analysis. At least 150 cells or colonies were scored in evaluations of phenotype, and figures are quoted as mean with standard deviation. Significance was assessed by the Student's $t$-test. Haploid cell suspensions were sonicated to facilitate cell counting.

\section{RESULTS}

\section{Generation of the new GDH3 mutants}

Deletion of an EcoRI fragment from the polyGThybridizing insert denoted pPZ34 (Fig. 1, pPZ34-3) resulted in unregulated starvation phenotypes in both haploid and diploid cells (described more fully below) and so a more detailed analysis of this clone was carried out. For convenience of in vitro manipulation, a new plasmid containing the pPZ34 insert was constructed (pUCZ34). This new plasmid consisted of the KpnI fragment, shown in Fig. 1, cloned into a derivative of pUC18 (YanischPerron et al., 1985) from which we had removed the single EcoRI site.

The EsoRI fragment of pPZ34 removed in our first deletion could be cut into two smaller fragments with the enzyme BstEII so, in the first two new deletions, the $U R A 3$ gene replaced each of these smaller fragments in turn (Fig. 1, pPZ34-1, pPZ34-2). In the last deletion, pPZ34-4, the UR $A 3$ gene replaced the EcoRV fragment indicated in Fig. 1. In a final construction, the $U R A^{3}$ gene was inserted into the BstEII site (Fig. 1, pPZ34-5).

The smallest deletion, which removed the polyGT tract (pPZ34-1), and the insertion into the BstEIl site (pPZ345), which increased the distance between the polyGT tract and neighbouring sequences, did not generate any new phenotypes. We concluded from these data that the new phenotypes were not associated with the polyGT tract and these clones were not studied further.

The deletions pPZ34-2 and pPZ34-4 both produced the same starvation phenotypes as the original EcoRI deletion (pPZ34-3, described below). DNA sequence analysis revealed that this clone corresponded to a region of chromosome I sequenced by Bussey et al. (1995) including the gene GDH3 (FUN51, YAL062W, GenBank accession no. U12980). There is a $22 \mathrm{bp}$ polyGT tract $61 \mathrm{bp}$ from the first codon of GDH3 (Fig. 1). The MGG3 transformant, produced using pPZ34-4, was named MGG3D34.

We were unable to re-transform MGG3-D34 with GDH3containing plasmids. We were, however, able to transform MGG3 with a multicopy GDH3-containing plasmid, and no new phenotypes arose. This suggested to us that the failure in transformation was an additional phenotype of MGG3-D34, rather than a toxic effect of additional GDH3 protein. 


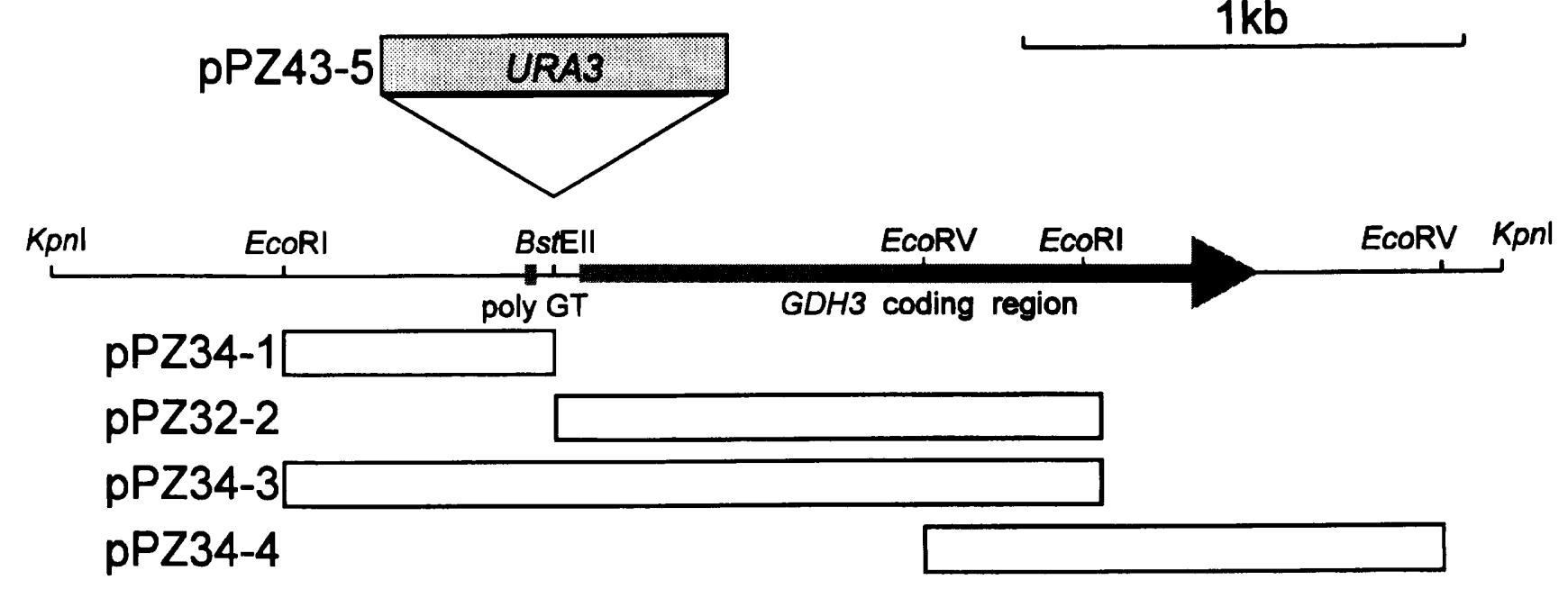

$1 \mathrm{~kb}$

Fig. 1. DNA fragments used in this study. The $3.32 \mathrm{~kb} \mathrm{Kpnl} \mathrm{fragment} \mathrm{illustrated} \mathrm{was} \mathrm{isolated} \mathrm{from} \mathrm{clone} \mathrm{pPZ34} \mathrm{described}$ in the text. Unfilled boxes below the map indicate the DNA fragments that were removed and replaced with the yeast URA3 gene. The box above the map indicates the insertion of the URA3 gene into the BstEIl site.
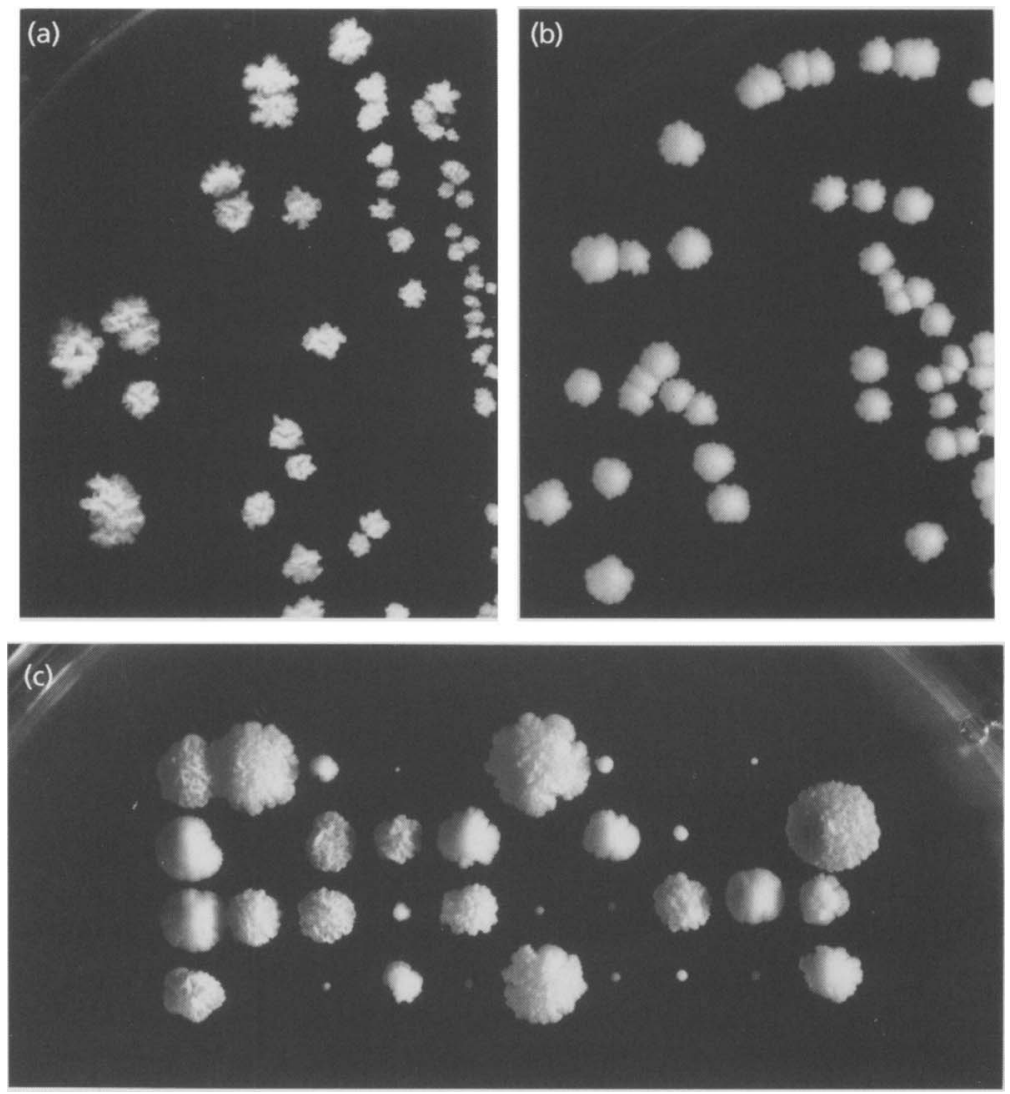

Fig. 2. Altered colony morphology in mutant strains. YEPD plates were incubated at $30^{\circ} \mathrm{C}$ for $6 \mathrm{~d}$. (a) Colonies from a restreaked haploid gdh3::URA3 mutant. (b) Colonies from a re-streaked wild-type isogenic haploid, SJR1Ya. (c) Ten individual dissections from the MGG3-D34 tetrads.

\section{Sporulation of the heterozygous GDH3/gdh3::URA3 diploid, MGG3-D34}

MGG3-D34 sporulated normally in 1\%(w/v) potassium acetate. Unusually however, $10 \%$ of cells grown to stationary phase in YEPG and $30 \%$ of cells grown to stationary phase in YEPA also sporulated. The parental diploid MGG3 produced less than $0.1 \%$ asci in parallel experiments. MGG3-D34 and the control strain MGG3 were then deliberately induced to sporulate on $1 \%$ potassium acetate and 20 of the resulting asci were dissected from each strain. Only four tetrads from MGG3D34 yielded four viable spores, the remainder yielded only two or three. Overall, half of the (viable) spores were $\mathrm{Ura}^{+}$and those which did not develop into colonies did not appear to have germinated. This pattern of spore 

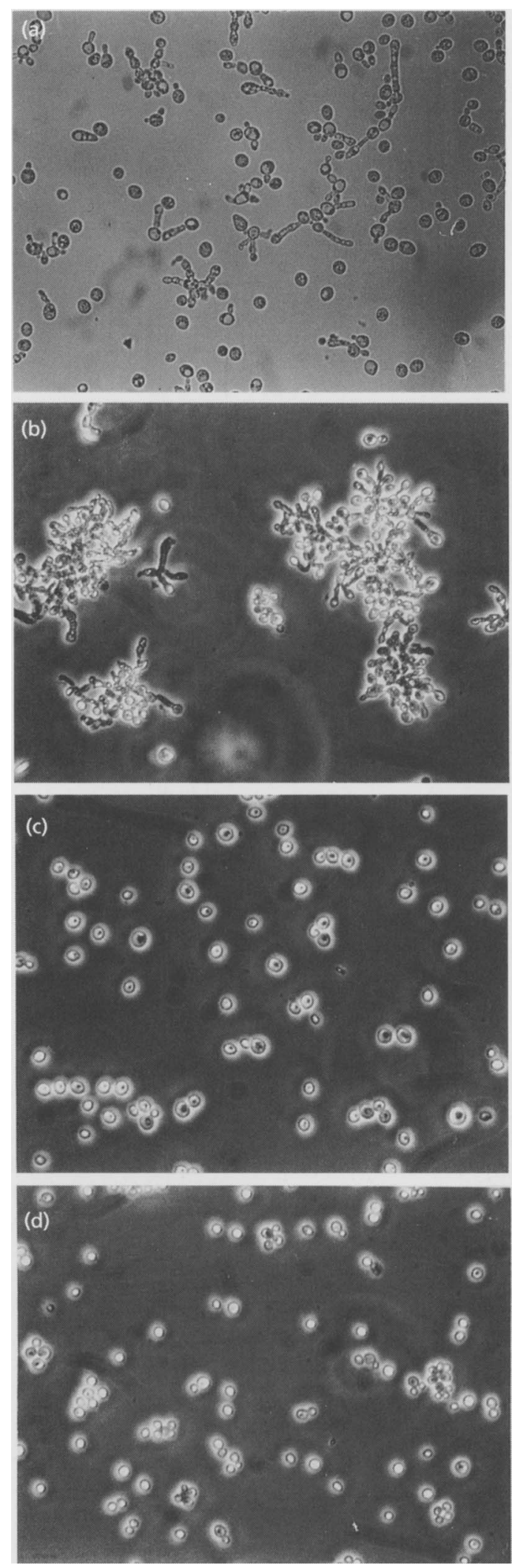

Fig. 3. Microscopic analysis of altered cell morphology in mutant strains. Cells from overnight cultures of (a) the diploid MGG3-D34, (b) the haploid gdh3:: URA3 mutant, (c) MGG3, and (d) a wild-type isogenic haploid, SJR1Ya. viability is consistent with random killing rather than the segregation of recessively lethal alleles. Many of the surviving haploids developed into very slow-growing colonies (Fig. 2). Only one spore failed to germinate in the control dissection (MGG3) and the rest developed into large normal colonies. Poor spore viability and germination in the MGG3-D34 dissection was not linked to the deletion. It seemed, therefore, that the mutation was interfering with some part of the sporulation process. We have called this an incompletely dominant meiotic lethal phenotype.

A more striking phenotype associated with the deletion was the development of a distinct wrinkled colony morphology often associated with dark brown colouring (Fig. 2). Microscopic analysis of cells from these aberrant colonies revealed the presence of a significant proportion $(38 \pm 5 \%)$ of abnormally growing cells which displayed either pseudohyphal or 'string-of-beads' cell types in which many of the cells displayed pronounced vacuolation (Fig. 3). After re-streaking the dark wrinkled clones, up to $5 \%$ of the resulting colonies (the proportion varied with different dark wrinkled spore clones) were either light and wrinkled or light and smooth.

\section{Outcrossing the new mutation}

Transformation has been shown to stimulate recombination and mutation (Danhash et al., 1991; Higgins \& Strathern, 1991). In order to ensure that these new mutant phenotypes were not a peculiarity of the MGG3 background, or the result of a second unlinked mutational event coincident with the disruption, two new types of diploid strain were constructed. The first type was produced by mating individual mutant (gdb3::UR $A 3$ ) $M A T \alpha$ spores to the haploid strain A364a, while the second (control) was produced by mating wild-type $M A T \alpha$ spores to A364a. Just as with cultures of the diploid MGG3-D34 (GDH3/gdh3:: UR A3), a high proportion $(20 \%)$ of cells in cultures from these new $G D H 3 / g d h 3:: U R A 3$ heterozygous diploids were able to sporulate on rich media. Less than $0.1 \%$ of the control GDH3/GDH3 strain sporulated under the same conditions. The new heterozygous diploids were sporulated by incubation in $1 \%$ potassium acetate and the resulting asci dissected. Just as the meiotic progeny of MGG3-D34, many of these spores failed to germinate. Surviving haploids which carried the deletion developed the aberrant colonial and cell morphology seen in MGG3-D34 segregants. These experiments confirm that the large $G D H 3$ deletion is responsible for the aberrant phenotypes. Homozygous gdh3::UR $A 3 / g d h 3:: U R A^{3}$ diploids, generated by mating mutant spores of opposite mating types, had the aberrant cell and colony morphology of the haploid mutants and, on sporulation, produced almost totally inviable progeny.

\section{How many yeast genes encode NADP-GDH-like proteins?}

The map position for $G D H 1$ has not yet been determined by tetrad analysis, but the high sequence homology 


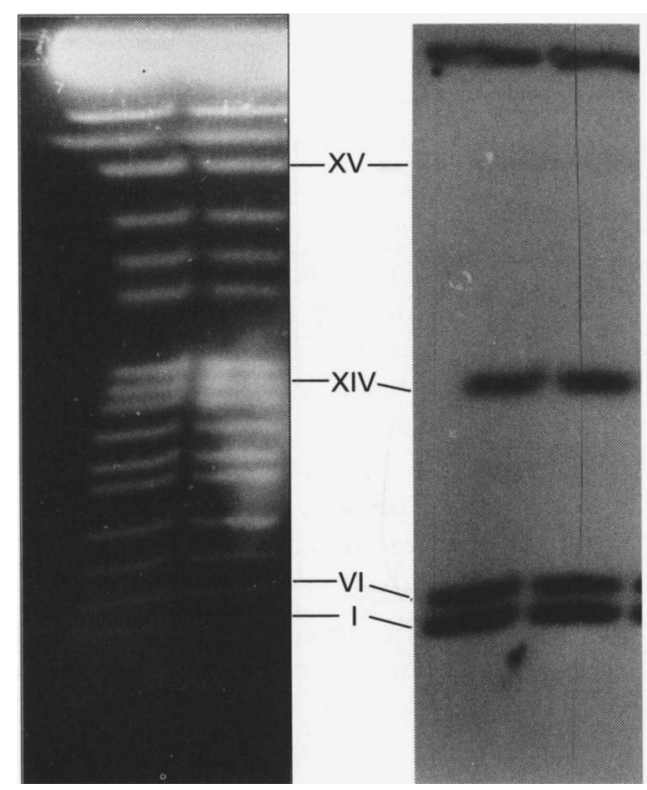

Fig. 4. Chromosomes which hybridize to the Kpnl fragment of chromosome I which contains GDH3. Left panel: separation of chromosomes of YP148 was achieved on a $10 \times 15 \mathrm{~cm}, 1 \%$ agarose gel at $135 \mathrm{~V}$ for $48 \mathrm{~h}$ with a switching interval of $145 \mathrm{~s}$. Right panel: following acid depurination, the gel was blotted and probed with the $3.32 \mathrm{~kb} \mathrm{Kpnl} \mathrm{fragment} \mathrm{(Fig.} \mathrm{1),} \mathrm{labelled} \mathrm{with}$ $[\alpha-32 \mathrm{P}] \mathrm{CTP}$.

between GDH1 and GDH3 allowed us to adopt the alternative approach to chromosome assignment. The $K p n I$ fragment of pPZ34 was used to probe a CHEF gel blot of the chromosomes of YP148 (Fig. 4). The probe hybridized strongly, not only to chromosome I, which carries GDH3, but also to chromosomes VI and XIV. There was also very faint hybridization to chromosome $\mathrm{XV}$. The GDH2 gene is on chromosome XII and the lack of hybridization to that chromosome confirms the lack of similarity between the sequences of GDH3 and GDH2. The complete sequence of chromosome VI has been released and only shares significant homology with our clone in two polyGT tracts. These are both $60 \mathrm{bp}$ long (the longest yet sequenced in yeast) and they probably account for the hybridization of our probe to this chromosome. This suggested to us that GDH1 is on chromosome XIV, and we are grateful to Werner Mewes for a search of the confidential yeast database at MIPS (Martinsried Institute for Protein Sequences, Max Planck Institute for Biochemistry, Munich, Germany) which confirms our finding.

\section{Nutritional dependence of mutant phenotypes}

Five haploid colonies from MGG3-D34 were further analysed. The pseudohyphal and wrinkled colony phenotypes developed on rich medium (YEP) supplemented with many different carbon sources $(0 \cdot 2,2$ or $10 \%, \mathrm{w} / \mathrm{v}$, glucose $; 2 \%, \mathrm{v} / \mathrm{v}$, glycerol $; 2 \%, \mathrm{w} / \mathrm{v}$, potassium acetate). On minimal medium (YNB plus ammonium sulphate), in contrast, aberrant colonies were never observed, although there was still a small proportion $(12 \pm 3 \%)$ of aberrant cell types. In order to determine whether this difference (significant at $P=0.05$ ) was a consequence of the different nitrogen source provisions in YNB and YEPD, cells were then plated onto media with additional or alternative supplements.

Colonies grown on YEPD supplemented with ammonium sulphate $(0.5 \%, \mathrm{w} / \mathrm{v})$ still had aberrant morphology and contained a high proportion of pseudohyphal cells ( $48 \pm 4 \%$; not significanlty different to YEPD alone). We conclude from this result that it was not simply the presence of ammonium ions in YNB which suppressed the mutant phenotype.

The deletion of $G D H 3$, by analogy with the phenotype of $g d b 1^{-}$mutants, might be expected to lead to a deficiency in glutamate and/or glutamine. To discover whether the phenotypes of gdh3::UR $A 3$ mutants had arisen as a consequence of this deficiency, colonies were plated on YEPD agar, supplemented $(2 \%, w / v)$ with these two amino acids. In fact, the proportion of aberrant cells actually increased slightly, though not significantly, on YEPD containing glutamine, either alone (to $54 \pm 6 \%$ ) or with glutamate (to $50 \pm 2 \%$ ). Glutamate alone had no effect $(35 \pm 5 \cdot 5 \%)$. Colonies were also grown on YNB agar containing glutamate $(0.2 \%)$ or glutamine $(0.2 \%)$, supplied either as an alternative to ammonium as nitrogen source or as an additional supplement. Again the addition of glutamine led to an increase in the proportion of aberrant cell types (compared to cells from YNB with ammonia alone), both as sole nitrogen source (to $41 \pm 3 \%$; significant at $P=0.01$ ), and with ammonia (to $55.6 \pm 1.9 \%$; significant at $P=0.01$ ), though the colonies remained wild-type in appearance (unwrinkled). We concluded from these experiments that starvation for glutamate or glutamine does not account for the mutant phenotype.

In all of the preceding experiments, adequate provision of nutrients led to starvation phenotypes. In order to assess the effects of genuine starvation, growing gdb3::UR $A 3$ haploid cells were patched onto YNB lacking either glucose or ammonia. After $10 \mathrm{~d}$, cells transferred to YEPD failed to grow. In a parallel experiment, the haploid SJR1Y $\alpha$ remained viable. This experiment suggests that, in $g d b 3:: U R A 3$ mutants, there is a general failure to interpret starvation signals properly.

\section{DISCUSSION}

A partial deletion of the second of two yeast genes encoding NADP-GDH (GDH3) has been constructed and results in a variety of aberrant starvation phenotypes. Haploid yeast strains carrying this allele, when grown on rich solid media, have crenulated colonies containing a high proportion $(38 \pm 5 \%)$ of pseudohyphal and other aberrant cell types. On poor media, such haploids quickly starve to death. Diploids, heterozygous for the deletion at the GDH3 locus, show a high level of sporulation on rich media and exhibit a partial meiotic lethality. 
Sporulation on rich media has been demonstrated previously in mutants with low cAMP levels. For example, cells carrying mutations in the adenylate cyclase gene, CYR1 (Matsumoto et al., 1985), or in two of its regulators, CDC25 (Broek et al., 1987) and RAS2 (Toda et al., 1985), also sporulate in rich media. In contrast, pseudohyphal growth is enhanced by the high cAMP levels induced by starvation for nitrogen, but not glucose (conditions which inhibit sporulation; Gimeno et al., 1992). Although the catabolic NAD-dependent glutamate dehydrogenase (encoded by $G D H 2$ ) has been shown to be regulated by the cAMP-dependent protein kinase A (Uno et al., 1984), we are not aware of any evidence for the similar regulation of NADP-GDH. It seems unlikely, therefore, that these contrasting mutant NADP-GDH phenotypes are a result of modulation of the RAS/cAMP pathway.

Until now, it has been supposed that gdb1 mutants were reliant on glutamine synthase for the fixation of ammonia and on glutamate synthase for the synthesis of glutamate. However, it is possible that, in gdb1 mutants, the GDH3 gene product can fulfil this role. Against this hypothesis there is biochemical evidence that NADP-GDH activity is lost in gdb1 mutants (Grenson \& Hou, 1972; Grenson et al., 1974). There are three NADP-GDH enzymes in Chlamydomonas reinhardtii which are induced under different trophic and stress conditions (Moyano et al., 1992) so it may be that the yeast genes too are differentially regulated. An alternative explanation for the lack of enzyme activity in the earlier study is that the strain concerned also carried at least one other pertinent mutation (per1; Magasanik, 1992) giving rise to other misleading phenotypes. It remains a formal possibility that the mutation named $g d h 1$ is not actually in the $G D H 1$ gene but instead affects a different gene which regulates all of the NADP-GDH genes. Since $G D H 3$ has been shown to produce a transcript in at least one strain (Howard Bussey, personal communication) and gdb1::UR $A 3$ mutants have a pronounced phenotype, we believe that the $G D H 3$ gene product has a distinct role in the cell.

It has been reported that yeast grown in nitrogen (ammonia)-limited chemostat culture produces aberrant, pseudohyphal cells (ter Schure et al., 1995a). The similar phenotype of gdh3::UR $A 3$ mutants suggests that they too might be nitrogen-starved or failing to sense nitrogen properly. Since most cellular nitrogen is derived from the amino group of glutamate, the simplest explanation for our data is that the GDH3 product has a real contribution to the fixed nitrogen pool. However, until the properties of this gene product have been assessed biochemically, the possibility remains that it is a component of the ammonia sensor proposed by ter Schure et al. (1995b). The fact that the addition of glutamine to YNB actually increases the proportion of aberrant cell types twofold suggests that nitrogen starvation alone cannot account for these new mutant phenotypes.

An alternative possibility is that, in these new mutants, nitrogen catabolite repression (Cooper, 1982; also called nitrogen regulation, Magasanik, 1992; Grenson, 1983) is occurring, leading to a more general starvation.
Unfortunately, attempts to address this possibility, by assaying the general amino acid permease (GAP) in various media, have been thwarted by the very clumpy growth of cultures in liquid media.

A last possibility, which arises from the work of Dickinson (1994), is that these mutants have a lesion in the metabolism of branched-chain amino acids. GDH1 is regulated by the Leu3 protein, a transcriptional regulator of branched-chain amino acid biosynthetic pathways ( $\mathrm{Hu}$ et al., 1995), and cells grown on ethanol, with leucine as a nitrogen source, produce long anucleate pseudohyphae (Dickinson, 1994). This possibility is to be investigated.

The partial meiotic lethal phenotype and the instability of the other phenotypes associated with these deletions are difficult to explain in terms of the effect on $G D H 3$ itself, though they may both be a consequence of translocations or other rearrangements of chromosome I. Two preliminary experiments support this hypothesis. First, the $G D H 3 / g d b 3:: U R A 3$, heterozygous diploids, produced by mating mutant spores to A364a, showed no significant increase in the rate of uncovering of the recessive markers ade 1 and ade 2 (data not shown) during mitotic growth, so the effect was probably not a consequence of chromosome loss. Second, in an analysis of chromosomes from several phenotypic variants of the haploid $g d h 3:: U R A 3$ strains we were rarely able to detect chromosome I, strongly suggesting recombinational activity. The apparent mobility of $\mathrm{Y}^{\prime}$ elements and SUC genes has suggested to some authors that DNA sequences close to telomeres are prone to rearrangement (Horowitz et al., 1984; Carlson et al., 1985), and, given the proximity of the GDH3 gene to a telomere, it may be that our modifications to this locus have induced instability in the region. We intend to investigate the fate of chromosome I more closely in these strains.

Starvation for nitrogen is an environmental trigger for sporulation in diploid cells and for foraging or arrest in haploid and diploid cells - the particular developmental fate being critically dependent on carbon source. It is important therefore that the cell can sense the difference between a poor nitrogen source and the lack of a nitrogen source in determining its developmental options. It may be that the product of $G D H 3$ has a role in that sensing pathway.

\section{ACKNOWLEDGEMENTS}

We thank David Parry, Stephen Mellor and Alistair Johnston for the time they have spent on some parts of this study, Howard Bussey for unpublished information concerning $G D H 3$, Mike Cherry for assistance with DNA sequence analysis, Werner Mewes for a search of the confidential yeast database at MIPS, Alan Eddy for advice on GAP assays and Steve Oliver for critically reading the manuscript.

\section{REFERENCES}

Altschul, S. F., Gish, W., Miller, W., Myers, E. W. \& Lipman, D. J. (1990). Basic local alignment search tool. J Mol Biol 215, 403-410.

Broek, D., Toda, T., Michael, T., Levin, L., Birchmeier, C., Zoller, M., Powers, S. \& Wigler, M. (1987). The S. cerevisiae CDC25 gene 
product regulates the RAS/adenylate cyclase pathway. Cell 48, 789-799.

Bussey, H., Kaback, D. B., Zhong, W., Vo, D. T., Clark, M. W., Fortin, N., Hall, J., Quellette, B. F. F., Keng, T., Barton, A. B., Su, Y., Davies, C. J. \& Storms, R. K. (1995). The nucleotide sequence of chromosome I from Saccharomyces cerevisiae. Proc Natl Acad Sci US A 92, 3809-3813.

Carlson, M., Celenza, J. L. \& Eng, F. D. (1985). Evolution of the dispersed SUC gene family by rearrangement of chromosome telomeres. Mol Cell Biol 5, 2894-2902.

Chu, G., Vollrath, D. \& Davis, R. W. (1989). Separation of large DNA molecules by contour clamped homogeneous electric fields. Science 234, 1582-1585.

Cooper, T. G. (1982). Nitrogen metabolism in Saccbaromyces cerevisiae. In The Molecular Biology of the Yeast Saccharomyces: Metabolism and Gene Expression, pp. 39-99. Edited by J. N. Strathern, E. W. Jones \& J. R. Broach. Cold Spring Harbor, NY: Cold Spring Harbor Laboratory.

Danhash, N., Gardner, D. C. J. \& Oliver, S. G. (1991). Heritable damage to yeast caused by transformation. Bio/Technology 9 , 179-182.

Dickinson, J. R. (1994). Irreversible formation of pseudohyphae by haploid Saccharomyces cerevisiae. FEMS Microbiol Lett 119, 99-103.

Gardner, D. C. J., Heale, S. M., Stateva, L. I. \& Oliver, S. G. (1993). Treatment of yeast cells with wall lytic enzymes is not required to prepare chromosomes for pulsed field gel analysis. Yeast 9, 1053-1055.

Gimeno, C. J., Ljungdahl, P. O., Styles, C. A. \& Fink, G. R. (1992). Unipolar cell divisions in the yeast $S$. cerevisiae lead to filamentous growth: regulation by starvation and RAS. Cell 66, 1077-1090.

Goebl, M. \& Petes, T. D. (1986). Most of the yeast genomic sequences are not essential for cell growth and division. Cell 46, 983-992.

Grenson, M. (1983). Study of the positive control of the General Amino Acid Permease and other ammonia sensitive uptake systems. Eur J Biochem 133, 141-144.

Grenson, M. \& Hou, C. (1972). Ammonia inhibition of the general amino acid permease and its suppression in NADP-specific glutamate dehydrogenaseless mutants of Saccharomyces cerevisiae. Biocbem Biopbys Res Comm 48, 749-758.

Grenson, M., Dubois, E., Piotrowska, M., Drillien, R. \& Aigle, M. (1974). Ammonia assimilation in Saccharomyces cerevisiae as mediated by the glutamate dehydrogenases: evidence for the gdba locus being the structural gene for NADP-dependent glutamate dehydrogenase. Mol Gen Genet 128, 73-85.

Higgins, D. R. \& Strathern, J. N. (1991). Electroporation-stimulated recombination in yeast. Yeast 7, 823-831.

Horowitz, H., Thorburn, P. \& Haber, J. E. (1984). Rearrangements of highly polymorphic regions near telomeres of Saccharomyces cerevisiae. Mol Cell Biol 4, 2509-2517.

Hu, Y., Cooper, T. G. \& Kohlhaw, G. B. (1995). The Saccharomyces cerevisiae Leu3 protein activates expression of GDH1, a key gene in nitrogen assimilation. Mol Cell Biol 15, 52-57.

Magasanik, B. (1992). Nitrogen utilization. In The Molecular and Cellular Biology of the Yeast Saccharomyces: Gene Expression, pp.
283-317. Edited by E. W. Jones, J. R. Pringle \& J. P. Broach. Cold Spring Harbor, NY: Cold Spring Harbor Laboratory.

Matsumoto, K., Uno, I. \& Ishikawa, T. (1985). Genetic analysis of the role of cAMP in yeast. Yeast 1, 15-24.

Moyano, E., Cardenas, J. \& Munoz-Blanco, J. (1992). Purification and properties of three $\mathrm{NAD}(\mathrm{P})^{+}$isozymes of $\mathrm{L}$-glutamate dehydrogenase of Chlamydomonas reinhardtii. Biochem Biopbys Acta 1119, 63-68.

Moye, W. S., Amuro, N., Rao, J. K. M. \& Zalkin, H. (1985). Nucleotide sequence of yeast GDH1 encoding nicotinamide dinucleotide phosphate-dependent glutamate dehydrogenase. J Biol Chem 260, 8502-8508.

Oliver, S. G. (1996). From DNA sequence to biological function. Nature 370, 597-600.

Rosenkrantz, M., Alam, T. M., Kim, K.-S., Clark, B. J., Srere, P. A. \& Guarente, L. P. (1986). Mitochondrial and non-mitochondrial citrate synthases in Saccharomyces cerevisiae are encoded by distinct homologous genes. Mol Cell Biol 6, 4509-4515.

Rosenkrantz, M., Kell, C. S., Pennell, E. A., Webster, M. \& Devenish, L. J. (1994). Distinct upstream activation regions for glucose-repressed expression of the yeast citrate synthase gene CIT1. Curr Genet 25, 185-195.

Sambrook, J., Fritsch, E. F. \& Maniatis, T. (1989). Molecular Cloning: a Laboratory Manual, 2nd edn. Cold Spring Harbor, NY: Cold Spring Harbor Laboratory.

ter Schure, E. G., Silljé, H. H. W., Raeven, L. J. R. M., Boonstra, J., Verkleij, A. J. \& Verrips, C. T. (1995a). Nitrogen-regulated transcription and enzyme activities in continuous cultures of Saccharomyces cerevisiae. Microbiology 141, 1101-1108.

ter Schure, E. G., Silljé, H. H. W., Verkleij, A. J., Boonstra, J. \& Verrips, C. T. (1995b). The concentration of ammonia regulates nitrogen metabolism in Saccharomyces cerevisiae. I Bacteriol 177, $6672-6675$.

Sherman, F., Fink, G. R. \& Hicks, J. B. (1982). Methods in Yeast Genetics. Cold Spring Harbor, NY: Cold Spring Harbor Laboratory.

Toda, T., Uno, I., Ishikawa, T., Powers, S., Kataoka, T., Broek, D., Cameron, S., Broach, J., Matsumoto, K. \& Wigler, M. (1985). In yeast, RAS proteins are controlling elements of adenylate cyclase. Cell 40, 27-36.

Uno, I., Matsumoto, K., Adachi, K. \& Ishikawa, T. (1984). Regulation of the NAD-dependent glutamate dehydrogenase by protein kinases in Saccharomyces cerevisiae. J Biol Chem 259,1288-1293.

Walmsley, R. M., Chan, C. S. M., Tye, B.-K. \& Petes, T. D. (1984). Unusual sequences associated with the ends of yeast chromosomes. Nature 310, 157-160.

Wilkinson, B. M. (1991). Analysis of the potential Z-DNA sequences of the yeast Saccharomyces cerevisiae. $\mathrm{PhD}$ thesis, University of Manchester Institute of Science and Technology.

Yanisch-Perron, C., Viera, J. \& Messing, J. (1985). Improved M13 phage cloning vectors and host strains: nucleotide sequences of M13mp18 and pUC19 vectors. Gene 33, 103-119.

Received 13 November 1995; revised 29 January 1996; accepted 2 February 1996. 